VER0

Preprint typeset using $\mathrm{LT}_{\mathrm{E} X} \mathrm{X}$ style emulateapj v. 11/10/09

\title{
SUNRISE/IMAX OBSERVATIONS OF CONVECTIVELY DRIVEN VORTEX FLOWS IN THE SUN
}

\author{
J. A. Bonet ${ }^{1,2}$, I. Márquez ${ }^{1,3}$, J. SÁnchez Almeida ${ }^{1,2}$, J. Palacios ${ }^{4}$, V. Martínez Pillet ${ }^{1}$, S. K. Solanki ${ }^{5,6}$, J. C. \\ del Toro Iniesta ${ }^{7}$, V. Domingo ${ }^{4}$, T. Berkefeld ${ }^{8}$, W. Schmidt ${ }^{8}$, A. Gandorfer ${ }^{5}$, P. Barthol $^{5}$, And M. Knölker ${ }^{9}$ \\ Draft version November 14, 2018
}

\begin{abstract}
We characterize the observational properties of the convectively driven vortex flows recently discovered on the quiet Sun, using magnetograms, Dopplergrams and images obtained with the 1-m balloon-borne SUNRISE telescope. By visual inspection of time series, we find some $3.1 \times 10^{-3}$ vortices $\mathrm{Mm}^{-2} \mathrm{~min}^{-1}$, which is a factor of $\sim 1.7$ larger than previous estimates. The mean duration of the individual events turns out to be $7.9 \mathrm{~min}$, with a standard deviation of $3.2 \mathrm{~min}$. In addition, we find several events appearing at the same locations along the duration of the time series (31.6 min). Such recurrent vortices show up in the proper motion flow field map averaged over the time series. The typical vertical vorticities are $\lesssim 6 \times 10^{-3} \mathrm{sec}^{-1}$, which corresponds to a period of rotation of some $35 \mathrm{~min}$. The vortices show a preferred counterclockwise sense of rotation, which we conjecture may have to do with the preferred vorticity impinged by the solar differential rotation.

Subject headings: convection — Sun: granulation — Sun: photosphere — Sun: surface magnetism
\end{abstract}

\section{INTRODUCTION}

Solar surface convection is driven by localized downdrafts that collect the cold plasma returning to the solar interior after releasing internal energy (e.g. Spruit et al. 1990; Stein \& Nordlund 1998; Rast 1998). Angular momentum conservation forces the plasma to spin up as it approaches the sinkhole, and vortices are formed at the downdrafts. Such convectively driven vortices were theoretically predicted and sought for long (e.g., Spruit et al. 1990; van Ballegooiien et al. 1998), but their observational discovery is fairly recent (Bonet et al. 2008; Wedemever-Böhm \& Rouppe van der Voort 2009; Balmaceda et al. 2010; Goode et al. 2010, with the wellknown earlier detection of a single large whirlpool by Brandt et al. 1988). In addition to supporting numerical models of solar surface convection, the photospheric vortices may be of importance as heating sources for the outer solar atmosphere. The downdrafts not only advect vorticity but also magnetic fields, which are intensified to $\mathrm{kG}$ field strengths in and around them. Buoyancy and the vertical geometry of the downdraft tend to align the magnetic field lines with the vertical, so that the spinning motions at photospheric levels can be propagated upward using the field lines as guides (e.g., Choudhuri et al.

\footnotetext{
${ }^{1}$ Instituto de Astrofísica de Canarias, E-38205 La Laguna, Tenerife, Spain

2 Departamento de Astrofísica, Universidad de La Laguna, E38071 La Laguna, Tenerife, Spain

${ }^{3}$ Departamento de Análisis Matemático, Universidad de La Laguna, E-38271 La Laguna, Tenerife, Spain

${ }^{4}$ Laboratorio de Procesado de Imágenes, Universidad de Valencia, E-46980 Paterna, Valencia, Spain

${ }^{5}$ Max Planck Institut für Sonnensystemforschung, Max Planck Strasse 2, Katlenburg-Lindau, 37191, Germany

${ }^{6}$ School of Space Research, Kyung Hee University, Yongin, Gyeongg 446-701, Republic of Korea

${ }^{7}$ Instituto de Astrofísica de Andalucía, Camino Bajo Huetor 50, 18008, Granada, Spain

${ }^{8}$ Kiepenheuer-Institut für Sonnenphysik, Schöneckstr. 6, D79110, Freiburg, Germany

${ }^{9}$ High Altitude Observatory, National Center for Atmospheric Research, P.O. Box 3000, Boulder CO 80307-3000, USA. (NCAR is sponsored by the National Science Foundation)
}

1993; Zirker 1993; van Ballegooijen et al. 1998). Waves thus excited transport photospheric energy that can be deposited in higher layers of the atmosphere. Moreover, downdrafts often trap structures of mixed polarity, so that the swirling motions wind up opposite polarity field lines, facilitating magnetic reconnection and the ensuing energy release.

These convectively driven vortex flows are a recently discovered phenomenon poorly characterized from an observational point of view. So far, we only know that the vortices are quite common, have no preferred sense of rotation at the solar equator, and last (at least) minutes (Bonet et al. 2008). They are also visible in the chromosphere, where they seem to be associated with significant blueshifts (Wedemeyer-Böhm \& Rouppe van der Voort 2009). Most of them are small-scale $(\lesssim 0.5 \mathrm{Mm}$ Bonet et al. 2008), but some have a much larger radius of influence (up to $20 \mathrm{Mm}$, Attie et al. 2009; Brandt et al. 1988). Lifetimes can be longer than $20 \mathrm{~min}$, and several observables (such as circular polarization and G-band intensity) simultaneously indicate the presence of vortical motions (Balmaceda et al. 2010). In terms of global properties rather than individual eddies, the vertical vorticity inferred from proper motions seems to be higher in downflow regions, suggesting excess vorticity in intergranular lanes (Wang et al. 1995; Pötzi \& Brandt 2005). Nisenson et al. (2003) searched for evidence of vorticity in the motions of isolated G-band bright points.

SunRISE is a 1-m balloon-borne solar telescope (Barthol et al. 2010) which, together with its Imaging Magnetograph eXperiment (IMaX, Martínez Pillet et al. 2010), provides time series with state-of-the-art high spatial resolution images and magnetograms. The dataset is ideal for a systematic characterization of the poorly known physical properties of the vortices. Thus this Letter presents a comprehensive observational characterization of the vortices in the photosphere. The actual data set and the procedure to detect vortices are described in $\S 2$. The main results are summarized in $\S 3$. Based on such results, we compare 
the observed properties with the predictions of the numerical simulations of solar surface convection ( $\S$ 4).

\section{OBSERVATION AND ANALYSIS PROCEDURE}

The data were gathered with IMaX near the solar disk center on June 9, 2009 (UT 01:31-02:02; although the exact location is not known) during the first science flight of SunRISE (Barthol et al. 2010; Solanki et al. 2010). The IMaX magnetograph uses a $\mathrm{LiNbO}_{3}$ etalon operating in double pass, liquid crystal variable retarders as the polarization modulator, and a beam splitter as the polarization analyzer. We use here data recorded in the so-called V5-6 observing mode (see Martínez Pillet et al. 2010) where images of the four Stokes parameters were taken at five wavelengths along the profile of the magneticsensitive line Fe I $\lambda 5250.2 \AA( \pm 80, \pm 40 \mathrm{~m} \AA$ from line center, plus continuum at $+227 \mathrm{~m} \AA$ ). After the science observing run a calibration set consisting of 30 in-focus and out-of-focus image-pairs was recorded for post-facto retrieval of the point spread function (PSF) using phase diversity (Gonsalves 1982; Paxman et al. 1996). The science images were reconstructed by deconvolution using a modified Wiener filter and the calibrated PSF of the optical system. IMaX provides $85 \mathrm{~m} \AA$ spectral resolution and between 0.15 and $00^{\prime \prime} 18$ angular resolution in the reconstructed images. Dopplergrams and magnetograms are derived from the Stokes parameters by using the approach described in (Martínez Pillet et al. 2010). All in all, the reduction procedure renders time series of images, magnetograms, and Dopplergrams with a cadence of $33 \mathrm{sec}$, a spatial sampling of $0^{\prime \prime} .055$, and an effective field-of-view (FOV) of $45^{\prime \prime} \times 45^{\prime \prime}$. As inferred from the standard deviation of the polarization signals at the continuum wavelength, the circular polarization noise is $5 \times 10^{-4}$ in units of the continuum intensity. The observing material analyzed here consists of a time series lasting $31.6 \mathrm{~min}$. Movies were generated after rigid alignment and $p$-mode subsonic filtering (Title et al. 1989).

Bonet et al. (2008) found the small-scale vortex flows by visual feature tracking of magnetic G-band bright points $(\mathrm{BPs})$. The same downdrafts producing vortices also advect and concentrate magnetic flux (see $\S$ 1), which often appears as BPs when the field strength is in the $\mathrm{kG}$ regime (see, e.g., Sánchez Almeida et al. 2004, and references therein). As Bonet and colleagues acknowledge, the technique is rather limited since whirlpools without BPs are expected, and they escape from detection. Taking advantage of the combined high spatial resolution and high polarimetric sensitivity of IMaX/SunRISE data, we tried to detect and study vortices in longitudinal magnetograms, which are sensitive not only to kGs fields, but to plasmas with the full range of field strengths. In addition, we broaden the study using continuum intensity, line minimum intensity, lineof-sight (LOS) velocity, and line width (the last three parameters obtained from a Gaussian fit to the five sampled wavelengths). Individual vortices are identified and characterized through the following steps:

(1) The detection is based on a visual inspection of longitudinal-magnetogram movies. Playing back and forth these movies, we identify those locations and time intervals where structures seem to rotate.

(2) Once a vortex candidate is thus located, it is iso- lated in a $5^{\prime \prime} .5 \times 5^{\prime \prime} .5$ sub-field, where the corresponding sub-fields in the other four physical parameters are visually inspected for swirling motions (see Fig. 1).

(3) Horizontal velocity maps of the event in all the five parameters are created from proper motions. The horizontal motions are measured in these reduced subfields employing the local correlation tracking (LCT) algorithm of November \& Simon (1988), as implemented by Molowny-Horas \& Yi (1994), and with a Gaussian tracking window of about 0 ". 4 FWHM. In order to help the algorithm, the original images are interpolated in time and space so as to have a pixel of $0 . \prime 028$ and a cadence of $11 \mathrm{sec}$. The horizontal velocities obtained by comparing successive images are time averaged the duration of the event. Examples of such velocity maps are shown in Fig. 2. The size of the tracking window was chosen as a trade off to be large enough for the LCT algorithm to have structures to track, yet small enough to minimize the presence of several structures with different velocities. If the velocity maps do not show a regular closed shape in at least two physical parameters, then we discard the vortex candidate and start from step 1.

(4) We compute the vertical vorticity, the divergence, and the curvature corresponding to the LCT horizontal velocities. Given the velocity $\mathbf{U}$, the vertical vorticity $(\nabla \times \mathbf{U})_{z}$ can be interpreted in terms of the local angular velocity since a plasma in pure rotational motion has $(\nabla \times \mathbf{U})_{z}=2 w$, with $w$ the angular velocity. Similarly, the curvature of such motion is $\kappa=\frac{1}{2}(\nabla \times \mathbf{U})_{z}|\mathbf{U}|^{-1}$, with $\kappa^{-1}$ the radius of curvature. Examples of vorticity and curvature maps are shown in Figs. 3, 4.

(5) Using the LCT horizontal velocities, we track the evolution of passively advected tracers (corks) spread out all over the sub-field (Yi 1992). As time goes by, the corks end up in the sinkhole, revealing the position of the sink according to different physical parameters (see the white points in Fig. 3). If the sinkhole positions inferred from the different physical parameters do not agree within 0 ". 4, then the vortex is discarded and we return to step 1.

We find the curvature maps to be an efficient complementary tool for vortex center detection. In most cases these maps show up the sinkholes as conspicuous pointlike features (see Fig. 4) with positions that agree well with the centers determined as the final destination of the corks in the corresponding movie (c.f. Figs. 3, 4).

The above list outlines the general procedure, but we do not disregard casual detections, e.g., when a second vortex was observed in any of the subfields corresponding to another vortex. Thus we find by chance some vortices which do not show up in the magnetogram signals. In addition to the LCT velocity maps of the individual vortices, we also computed the flow field for the full FOV during the full duration of the sequence. Isolated pointlike features in the corresponding curvature map suggest the presence of vortices, and their existence motivates further inspection of magnetograms for swirling motions. As one can image from this cumbersome procedure, the FOV has been unequally searched. We focused on those regions were the magnetograph signals were largest, so that the effective FOV of our research is only $28 . .5 \times$ $28 . .5$. This area is used in the estimates below unless otherwise stated.

Two animations, one showing the event in Figs. 1, 4 
and another with a different one, are given in the on-line material in the electronic edition of the Journal.

\section{RESULTS}

Following the procedure outlined above, we detected 42 vortices with proper velocity maps. They imply a spacetime-density of $d \simeq 3.1 \times 10^{-3}$ vortices $\mathrm{Mm}^{-2} \mathrm{~min}^{-1}$. In addition, 31 structures showing vortical motion were discarded because they did not fullfill our strict selection criteria. The selected events are used here to characterize the observational properties of vortices.

We have assigned a duration to each vortex, computed as the time interval in which the vortex motions are clearest. These durations span from 5 min to 20 min with a mean of $\tau \simeq 7.9 \mathrm{~min}$ and a standard deviation of $3.2 \mathrm{~min}$. The interpretation of these intervals as life-times is not devoid of uncertainty, though. Often we shorten the interval to assure a most pure swirling motion. In addition, some vortices appear in the mean flow field corresponding to the full time series, indicating that they probably last longer than the time span of the series itself. These long lasting vortices often involve a complex behavior: several short-lived vortices appear and disappear in the same location, giving rise to recurrent vortices. The position of their vortex centers may be static or drift with time. The recurrent vortices may or may not keep the same sense of rotation. In the latter case, however, we cannot consider the vortices to be strictly recurrent. We even find cases where the presence of a vortex is hinted as a clear pointlike feature in the curvature image, but we failed to identify any vortex at that position during the sequence.

The largest surprise of our analysis is the finding of a preferred sense of rotation: 27 counterclockwise vs. 15 clockwise. This is a big difference with respect to Bonet et al. (2008), where the two senses of rotation were observed equally. We analyze this issue in $\S 4$

Figure [5 shows histograms of vertical vorticities to characterize our measurements. The solid line corresponds to the vorticity in a region $2 \mathrm{Mm}$ wide around a number of well defined vortices. The histogram considering only $0.5 \mathrm{Mm}$ is shown as the dotted-dashed line. These histograms reveal the signature of the vortices, which turn out to have vorticities up to $0.006 \mathrm{sec}^{-1}$, corresponding to a period of rotation of some $35 \mathrm{~min}$. These are vorticities inferred from the magnetograms, which are systematically smaller than those obtained from the other observables. The difference can be pinned down to the proper motion velocity field, which tends to zero outside the large magnetic concentrations, where the polarization signals are low. The proper motions inferred from the other parameters extend throughout (Fig. 2), so that the histograms of vorticities have less contribution at low values and show extended tails (cf. the dashed line and the dotted line in Fig. 5, which represent the histograms of vorticities for the full FOV inferred from the magnetogram and the continuum intensity, respectively).

If a vortex has its axis tilted with respect to the LOS, it should produce a characteristic Doppler signal similar to the rotation curve of a galaxy, with a close pair redshiftblueshift centered at the sinkhole. The expected signals are of the order of a few hundred $\mathrm{m} \mathrm{s}^{-1}$ for moderatehigh inclinations $\left(30^{\circ}\right)$, which are at the limit of our ob- servation. We unsuccessfully seek for such signals in the Doppler maps, meaning that the vortex motions are not highly tilted with respect to the horizontal plane. Moreover, we note the discovery of horizontal vortex flows near the edges of granules reported by Steiner et al. (2010) using the same SunRISE/IMaX data and that are likely to be of a different nature to those analyzed here.

\section{DISCUSSION}

Small-scale vortex flows in quiet Sun are detected using five different observational parameters: magnetogram, continuum intensity, line core intensity, LOS velocity, and line width. The fact that in most cases the detection is consistent in three or more of these observables (showing different morphology) reinforces the reliability of the events found.

The number density of vortices is $\sim 1.7$ times larger than that found by Bonet et al. (2008), even though we have been far more strict here. The increase can be ascribed to the use of a larger variety of physical parameters to detect the swirls. Most of the vortices are shortlived events observed during less than $10 \mathrm{~min}$, but some of them last longer than the full time series, with recurrent vortices appearing in roughly the same place.

Vortices have a typical vorticity smaller than $6 \times 10^{-3} \mathrm{sec}^{-1}$, which corresponds to a period of rotation of some $35 \mathrm{~min}$. For reference, the large maelstrom found by Brandt et al. (1988) had a vorticity ten times smaller, with an associated period of some 6 hours. The measured vorticities are generally much smaller than those predicted by the numerical simulations of magneto convection (Stein \& Nordlund 1998, Stein 2010 private communication). We think that the bulk of this difference can be attributed to the limited spatial-temporal resolution of the observations. We are unable to identify vortices smaller than the tracking window, and/or lasting less than 8-10 frames, which sets an upper limit to the vorticity of some $0.04 \mathrm{sec}^{-1}$. Simulations indicate that the highest vorticities occur at the smallest resolvable scales and, thus, the predicted distribution critically depends on the numerical resolution of the simulation (see Fig. 31 in Stein \& Nordlund 1998).

The curvature maps (i.e., maps of the inverse radius of curvature) show the presence of vortices much better than the vorticity maps (see Figs. 3, 4). The vorticity is sensitive to the flow speeds, which are large outside vortices, creating spurious vorticity signals. The curvature, however, only enhances areas where the swirling motions occur at small scales, independently of the velocity. The sinkholes show up conspicuously as local extremes in the curvature maps, and this new property should be employed when devising automatic algorithms to detect vortices.

We find a preferred sense of rotation for the vortices (27 counterclockwise vs 15 clockwise). If the two senses of rotation were equally-probable then our observation would be highly unlikely (the probability is $4.4 \%$ assuming a binomial distribution). Nevertheless, the statistics is not large enough to provide a firm conclusion. The role of Coriolis forces on setting up this difference can also be discarded since the vortex motions involve time scales much too short to be affected by the solar rotation. The preferred sense of rotation may have to do with the solar differential rotation. The plasma pole- 


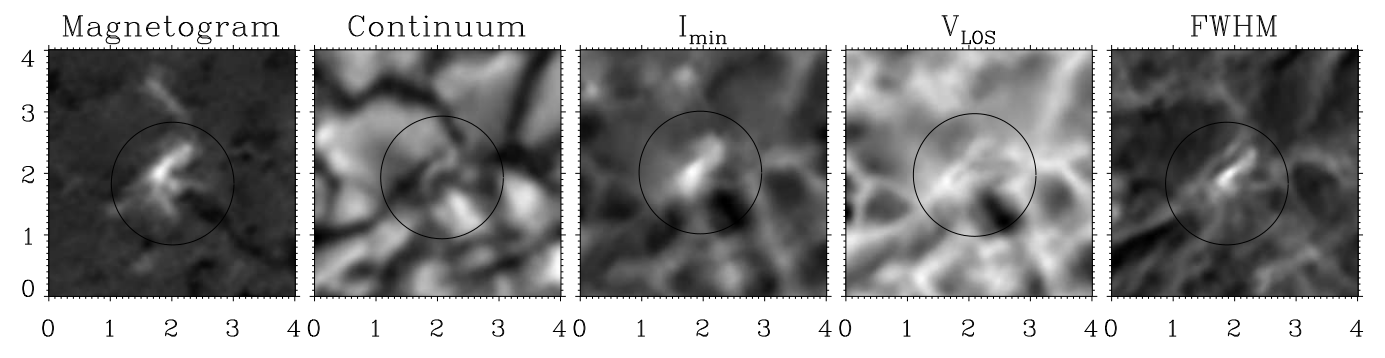

FIG. 1. - A particular vortex as reflected in different observational parameters - from left to right: magnetogram, continuum intensity, line core intensity, LOS velocity, and line width. The panels show the average along the life-time of the event $(\sim 6.7$ min), for every parameter. Horizontal scales are given in $\mathrm{Mm}$. The $1 \mathrm{-Mm}$ radius circles centered in the sinkhole are included for reference.

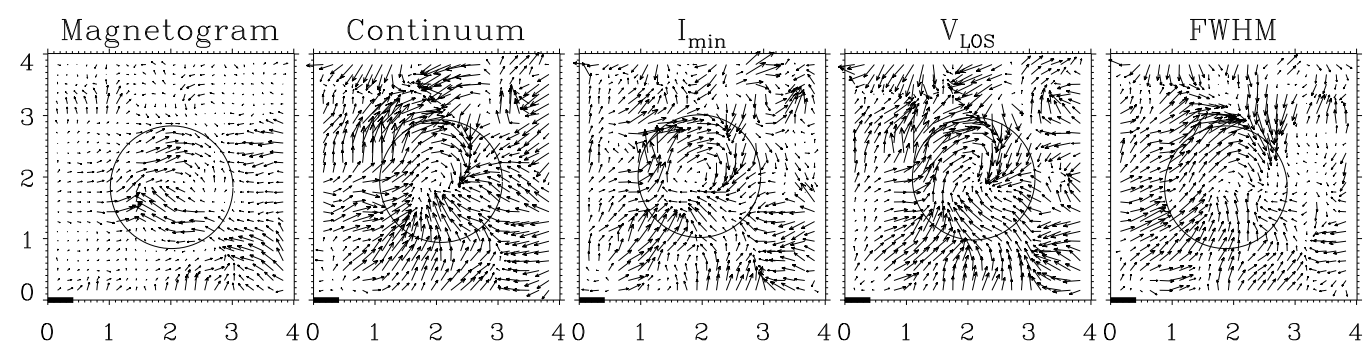

FIG. 2. - Horizontal velocity maps derived by LCT, from the proper motions of the parameters shown in Fig.1. The velocities are averaged over the life-time of the event $(\sim 6.7 \mathrm{~min})$. Horizontal scales are given in $\mathrm{Mm}$. The length of the black bar at coordinates $(0,0)$ corresponds to $1.8 \mathrm{~km} \mathrm{~s}^{-1}$.

The 1 -Mm radius circles centered in the sinkhole are included for reference.

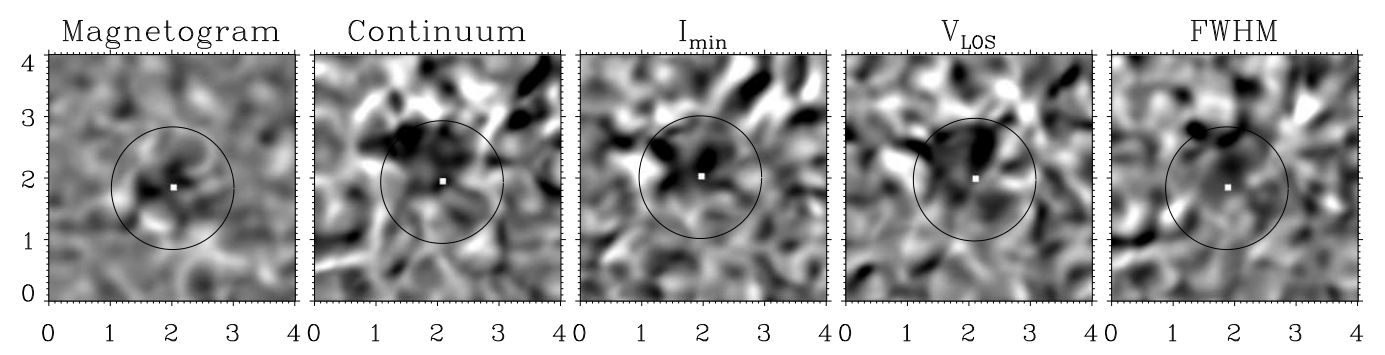

FIG. 3.- Maps of vertical vorticity corresponding to the LCT horizontal velocities for the parameters shown in Fig.1. The white points indicate the final position of the corks in the cork movies (see text). Horizontal scales are given in Mm. The 1 -Mm radius circles centered in the sinkhole are included for reference. Vorticities are represented in the range $\pm 6 \times 10^{-3} \mathrm{~s}^{-1}$, using a common grayscale in all the panels.

ward from the sink tends to lag behind, whereas the plasma equatorward from the sink moves forward. Such a difference impinges a preferred counterclockwise sense of rotation in the northern hemisphere, and a clockwise sense in the southern hemisphere. Back-of-the-envelope estimates indicate that the effect produces the right order of magnitude vorticity 10 If this conjecture turns out

10 Assume plasma separated by $40 \mathrm{Mm}$ (i.e., the size of a supergranule) and converging to a sinkhole in the middle. Then the difference of differential rotations at latitude $30 \mathrm{deg}$ produces a difference of velocities of some $35 \mathrm{~m} \mathrm{sec}^{-1}$. If the circulation is to be correct, it naturally explains the difference with respect to Bonet et al. (2008), whose observations correspond to the solar equator where there is no preferred sense of rotation. In our case the dominant counterclockwise rotation is consistent with an observed FOV in the northern hemisphere.

Thanks are due to R. Stein for discussions on the approximately conserved during the convergence, a vortex $0.5 \mathrm{Mm}$ wide would have a vorticity of $0.01 \mathrm{sec}^{-1}$. 


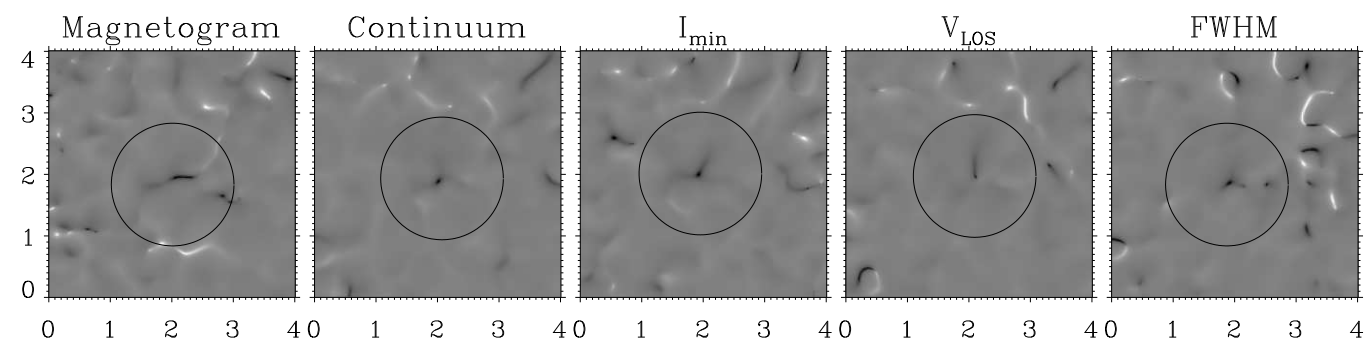

FIG. 4.- Curvature maps derived from the LCT horizontal velocities for the parameters shown in Fig.1. Horizontal scales are given in $\mathrm{Mm}$. The $1 \mathrm{-Mm}$ radius circles centered in the sinkhole are included for reference. Note how the curvatures clearly show the position of the sinkhole as an intense point-like feature. Curvatures are represented in the range $\pm 5 \times 10^{-2} \mathrm{~km}^{-1}$, using a common grayscale in all the panels.

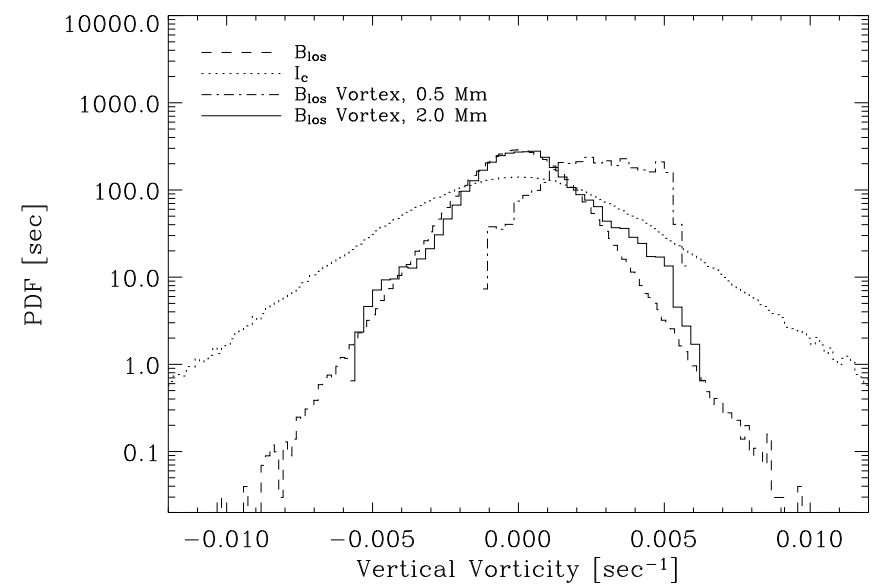

FIG. 5.- Probability density function (PDF) of vertical vorticities obtained from LCT proper motions. The 5 min time averaged distributions for the full FOV are shown as a dotted line, and a dashed line, depending on whether they were derived from the continuum intensity or the magnetogram, respectively. The dotted-dashed line corresponds to the vorticity in a region $0.5 \mathrm{Mm}$ wide around a number of well defined vortices (with the vorticity signed so that all vortices have positive vorticity). The solid line also represents a local histogram considering a region of $2 \mathrm{Mm}$. The excess of vorticity at some $0.005 \mathrm{sec}^{-1}$ is produced by the vortices. comparison with numerical simulations, and to C. Pastor for her support during the data interpretation. The German contribution to SUNRISE is funded by the Bundesministerium für Wirtschaft und Technologie through Deutsches Zentrum für Luft- und Raumfahrt e.V. (DLR), Grant No. 50 OU 0401, and by the Innovationsfond of the President of the Max Planck Society $(\mathrm{MPG})$. The Spanish contribution has been funded by the Spanish MICINN under projects ESP2006-13030C06, AYA2009-14105-C06 (including European FEDER funds), AYA2007-66502, AYA2007-63881 and by the EC (SOLAIRE Network - MTRN-CT-2006-035484). The HAO contribution was partly funded through NASA grant number NNX08AH38G. This work has been partly supported by the WCU grant (No R31-10016) funded by the Korean Ministry of Education, Science \& Technology Facilities: SUNRISE/IMAX

\section{REFERENCES}

Attie, R. Innes, D. E., \& Potts, H. E. 2009, A\&A, 493, L13

Balmaceda, L., Vargas Domínguez, S., Palacios, J., Cabello, I., \& Domingo, V. 2010, A\&A, 513, L6

Barthol, P., Gandorfer, A., Solanki, S. K. et al. 2010, Sol. Phys., in press (arXiv:1009.2689)

Bonet, J. A., Márquez, I., Sánchez Almeida, J., Cabello, I., \& Domingo, V. 2008, ApJ, 687, L131

Brandt, P. N., Scharmer, G. B., Ferguson, S., et al. 1988, Nature, 335,238

Choudhuri, A. R., Auffret, H., \& Priest, E. R. 1993, Sol. Phys., 143, 49, revise

Gonsalves, R. A. 1982, Optical Engineering, 21, 829

Goode, P. R., Yurchyshyn, V., Cao, W., et al. 2010, ApJ, 714, L31

Martínez Pillet, V., del Toro Iniesta, J. C., Álvarez-Herrero, A., et al. 2010, Sol. Phys., in press (arXiv:1009.1095)

Molowny-Horas, R. \& Yi, Z. 1994, Internal Rep. 31,Institute of Theoretical Astrophysics, University of Oslo, Oslo

Nisenson, P., van Ballegooijen, A. A., de Wijn, A. G., \& Sütterlin, P. 2003, ApJ, 587, 458

November, L. J. \& Simon, G. W. 1988, ApJ, 333, 427

Paxman, R. G., Seldin, J. H., Löfdahl, M. G., Scharmer, G. B., \& Keller, C. U. 1996, ApJ, 466, 1087

Pötzi, W. \& Brandt, P. N. 2005, Hvar Observatory Bulletin, 29, 61
Rast, M. P. 1998, Journal of Fluid Mechanics, 369, 125

Sánchez Almeida, J., Márquez, I., Bonet, J. A., Domínguez Cerdeña, I., \& Muller, R. 2004, ApJ, 609, L91

Solanki, S. K., Barthol, P., Danilovic, S., Feller, A. et al. 2010, ApJ, this issue

Spruit, H. C., Nordlund, Å., \& Title, A. M. 1990, ARA\&A, 28, 263

Stein, R. F. I. \& Nordlund, A. 1998, ApJ, 499, 914

Steiner, O., Franz, M., Bello González, N., Nutto, Ch. et al. 2010, ApJ, this issue

Title, A. M., Tarbell, T. D., Topka, K. P., et al. 1989, ApJ, 336, 475

van Ballegooijen, A. A., Nisenson, P., Noyes, R. W., et al. 1998, ApJ, 509, 435

Wang, Y., Noyes, R. W., Tarbell, T. D., \& Title, A. M. 1995

ApJ, 447, 419

Wedemeyer-Böhm, S. \& Rouppe van der Voort, L. 2009, A\&A, 507, L9

Yi, Z. 1992, PhD thesis, University of Oslo, Oslo

Zirker, J. B. 1993, Sol. Phys., 147, 47 